\title{
Development of a Carrier Free Dry Powder Inhalation Formulation of Ketotifen for Pulmonary Drug Delivery
}

Authors

Fariba Azari ${ }^{1}$, Saeed Ghanbarzadeh², ${ }^{3}$, Rezvan Safdari ${ }^{4}$, Shadi Yaqoubi ${ }^{5}$, Khosro Adibkia ${ }^{6}$, Hamed Hamishehkar ${ }^{4}$

Affiliations

1 Faculty of Pharmacy, Tabriz University of Medical Sciences, Tabriz, Iran

2 Cancer Gene therapy Research Center, Zanjan University of Medical Sciences, Zanjan, Iran

3 Zanjan Pharmaceutical Nanotechnology Research Center, Zanjan University of Medical Sciences, Zanjan, Iran

4 Drug Applied Research Center, Tabriz University of Medical Sciences, Tabriz, Iran

5 Research Center for Evidence Based Medicine and Students' Research Committee, Tabriz University of Medical Sciences, Tabriz, Iran

6 Biotechnology Research Center and Department of Pharmaceutics, Faculty of Pharmacy, Tabriz University of Medical Sciences, Tabriz, Iran

Key words

Dry powder inhaler, DPI, Ketotifen, Pulmonary drug delivery, next generation impactor

$\begin{array}{ll}\text { received } & 04.03 .2018 \\ \text { accepted } & 23.06 .2018\end{array}$

Bibliography

DOI https://doi.org/10.1055/a-0649-0814

Published online: 18.9 .2019

Drug Res 2020; 70: 26-32

(c) Georg Thieme Verlag KG Stuttgart · New York

ISSN 2194-9379

\section{Correspondence}

Hamed Hamishehkar

Drug Applied Research Center

Tabriz University of Medical Sciences

Golgasht Ave

517838695 Tabriz

Iran

Tel.: + 98/41/33355 965, Fax: + 98/41/33346977

hamishehkar.hamed@gmail.com; Hamishehkarh@tbzmed.ac.ir

\section{ABSTRACT}

Background Pulmonary drug delivery route is gaining much attention because it enables to target the active ingredients directly to lung both for local and systemic treatments, which maximize the therapeutic effect and minimize unwanted systemic toxicity. Dry powder inhaler (DPI) systems for asthma therapy have shown several merits to the other pulmonary delivery systems such as nebulizers and metered dose inhalers. Purpose The present study aims to develop and optimize a DPI formulation for Ketotifen fumarate through spray drying technique.

Methods Particles size and morphology, crystallinity, and drug-excipient interaction of fabricated DPI formulations were evaluated by scanning electron microscopy, X-ray diffraction (XRD), differential scanning calorimetry (DSC), and Fourier Transform Infrared Spectroscopy methods, respectively. The aerosolization indexes and aerodynamic properties of dry powders were determined by next generation impactor. The powder flowability was assessed by measuring the Hausner ratio and compressibility index.

Results Among solvent systems, ethanol-water mixture produced the most desirable powder property for inhalation after spray drying. Although co-spray dried formulations with ammonium bicarbonate resulted in the porous structure, it was not beneficial for DPI formulations due to the interaction with Ketotifen. DSC and XRD experiments proved the amorphous structure of prepared powders, which were stable for 12 months.

Conclusion The results of this study demonstrate the potential of Ketotifen DPI formulation and pave a way to use it easily in an industrial scale.

\section{Introduction}

Numerous approaches have been employed for the treatment of the respiratory diseases. Direct delivery of the therapeutic compounds to the respiratory tract is currently considered to be the most promising method for directly relieving or treating respiratory diseases such as asthma. Compared with other methods, there are some unique advantages to this type of drug delivery such as the quick onset of action, small doses required by avoiding the first- 
pass metabolism, and reduced systemic doses, which lead to maximum therapeutic efficiency and minimum side effects, respectively. Furthermore, lower dosage regimens provide considerable cost saving especially with expensive therapeutic agents and drug with low potency [1-3]. Pressurized metered-dose inhalers (MDI), nebulizers, and dry powder inhalers (DPI) are three main delivery systems used for aerosol inhalation in humans. Because of advantages such as being propellant-free, portable, easy to operate, and low-cost devices with improved stability of the formulation as a result of the dry state, DPI appears to be the most promising formulation for pulmonary drug delivery [4, 5]. Spray drying has been considered as a simple one-step process for producing small particles for pulmonary administration in DPI format that can be easily scaled up [6-11]. Ketotifen, a tricyclic benzocycloheptathiophene derivative, has several properties suggesting that it might be useful in the management of asthma. This drug inhibits passive cutaneous anaphylaxis and has a mast cell stabilizing effect. Orally taken Ketotifen undergoes severe first pass metabolism and therefore less than $50 \%$ bioavailability has been reported for orally administered Ketotifen [12,13]. Furthermore, Ketotifen shows dosedependent drowsiness that even causes the break of drug administration by patients $[14,15]$. To provide a dosage form of Ketotifen with more efficiency and less side effect than traditional orally administered tablets, DPI formulation and pulmonary drug delivery seem to be very promising. To the best of our knowledge, only liposomal Ketotifen was developed as an inhalable dry powder inhalation formulation of Ketotifen [16, 17]. Ketotifen fumarate has enough solubility to be easily dissolved in the surfactant enriched bronco alveolar fluid. In addition, because of high-dose administration of Ketotifen (in mg level) the liposomal formulation would not be able to carry effective and required amounts of Ketotifen to the deep parts of the lung. Furthermore, particle aggregation is the main drawback of the solid state of lipid-based carriers such as liposomes $[18,19]$. Therefore, the primary aim of this study was to prepare a carrier-free Ketotifen DPI formulation for pulmonary delivery via an industrial and easily scaled-up approach.

\section{Materials and Methods}

\section{Materials}

Ketotifen fumarate was provided from Sifavitor S.P.A Company (Milan, Italy). HPLC grade acetonitrile was purchased from Duksan Pure Chemicals (Ansan, Korea). Ethanol and ammonium bicarbonate were supplied from JATA (Arak, Iran) and Sigma (Saint Louis, MO, USA) Companies, respectively.

- Table 1 Spray drying condition of prepared formulation.

\section{Spray drying procedure}

Ketotifen $(500 \mathrm{mg}$ ) was dissolved in $50 \mathrm{~mL}$ of solvent (water, ethanol, and water: ethanol mixture $(1: 1 \mathrm{v} / \mathrm{v}))$ and the solutions were spray dried using a mini spray dryer (DORSAtech, Iran) at different inlet temperature and peristaltic pump, aspiration rate of $70 \%$, and flow rate of $7 \mathrm{~mL} / \mathrm{min}$ ( $\triangleright$ Table 1 ). Immediately after powder collection from the product collection vessel of spray dryer, powder was packed into the tightly closed container and desiccated over silica gel at room temperature until further studies. Ammonium bicarbonate was also added to some of formulations to increase the quality of powder for aerosolization purposes.

\section{Powder characterization}

The shape and surface morphology of the particles were studied by the scanning electron microscope (MIRA3 TESCAN, Brno, Czech Republic) using image analysis software (Image-Pro Plus 4.5; Media Cybernetics, Silver Spring, MD). Differential scanning calorimetry (DSC-60, Shimadzu, Kyoto, Japan), calibrated using indium, was employed to assess enthalpy and melting points of the formulations. Samples $(5 \mathrm{mg})$ were heated in the range of $25-210{ }^{\circ} \mathrm{C}$ at a scanning rate of $10{ }^{\circ} \mathrm{C} / \mathrm{min}$ in aluminum pans under nitrogen gas. X-ray diffraction (XRD, D500, Siemens, Karlsruhe, Germany) Fourier Transform Infrared Spectroscopy (FTIR) (MB-100, Bomen, Canada) was used to investigate the possible drug-excipient interaction. The potassium bromide discs were prepared by compressing the powders at pressure of 15 tons for $10 \mathrm{~min}$ in hydraulic press. Scans were obtained at a resolution of $2 \mathrm{~cm}^{-1}$, from 4000 to $400 \mathrm{~cm}^{-1}$. A helium pycnometer (Quantachrome Instruments, Boynton Beach, FL, USA) was used to determine true densities (TD) of the powders (The mean value of triplicate determinations is reported). Approximately one gram of each powder sample was used after calibration of the instrument using standard stainless steel spheres supplied by the manufacturer. The flowability of powders were determined by Hausner ratio (HR) and compressibility index (Cl) according to the following equations:

$\mathrm{Cl}=\frac{\rho \mathrm{t}-\rho \mathrm{b}}{\rho \mathrm{t}}$

$H R=\frac{\rho t}{\rho b}$

The tapped volume was determined after tapping the cylindrical measure several times until a constant volume was achieved.

\section{In vitro aerosolization assessment}

The in vitro aerodynamic parameters including aerodynamic diameter, aerosolization efficiency, dispersion and deposition charac-

\begin{tabular}{|l|l|c|c|c|c|}
\hline Formulation code & Solvent & Ammonium Bicarbonate $(\mathbf{m g})$ & Inlet Temp $\left({ }^{\circ} \mathbf{C}\right)$ & Outlet Temp $\left({ }^{\circ} \mathbf{C}\right)$ & 80 \\
\hline F1 & Water & - & 120 & 80 & 3 \\
\hline F2 & Water:ethanol & - & 120 & 60 & 1 \\
\hline F3 & Ethanol & - & 70 & 75 & 1 \\
\hline F4-1 & Water & 500 & 120 & 75 & 3 \\
\hline F4-2 & Water & 2000 & 120 & 75 & 3 \\
\hline F4-3 & Water & 5000 & 120 & 3 \\
\hline
\end{tabular}


teristics of Ketotifen spray-dried powders were assessed by an Aerolizer ${ }^{\circledR}$ connected to the next generation impactor (NGI) with pre-separator and USP induction port (Copley Scientific, Nottingham, UK). The NGI was assembled and operated in accordance with USP General Chapter 601 to assess the drug delivered [20]. An appropriate number of hard gelatin capsules $(n=6)$ were filled with $20 \mathrm{mg}$ of spray dried powder. To ensure efficient particle capture and prevent inter-stage losses due to particle bounce, the particle collection surface of each stage was coated with Tween ${ }^{\circledR} 80$. For this purpose, every eight collection cups of the NGI were soaked into Tween ${ }^{\circledR} 80$ ethanolic solution (1\%) and placed under the fume hood until the complete evaporation of ethanol. The cups were placed into the apertures in the cup tray and the cup tray was located into the bottom frame and lowered into place. The impactor lid was closed with the sealed body attached and the handle was operated to lock the impactor together. Capsules were gathered after actuation for the study of remained powder. The induction port was connected to the first stage of the NGI. The flow rate was calibrated using a flow meter (DFM 2000, Copley Scientific, Nottingham, UK) and fixed at $60 \mathrm{~L} / \mathrm{min}$. Fine particle fraction (FPF), mass median aerodynamic diameter (MMAD), and geometric standard deviation (GSD) indexes were calculated using the Cop-
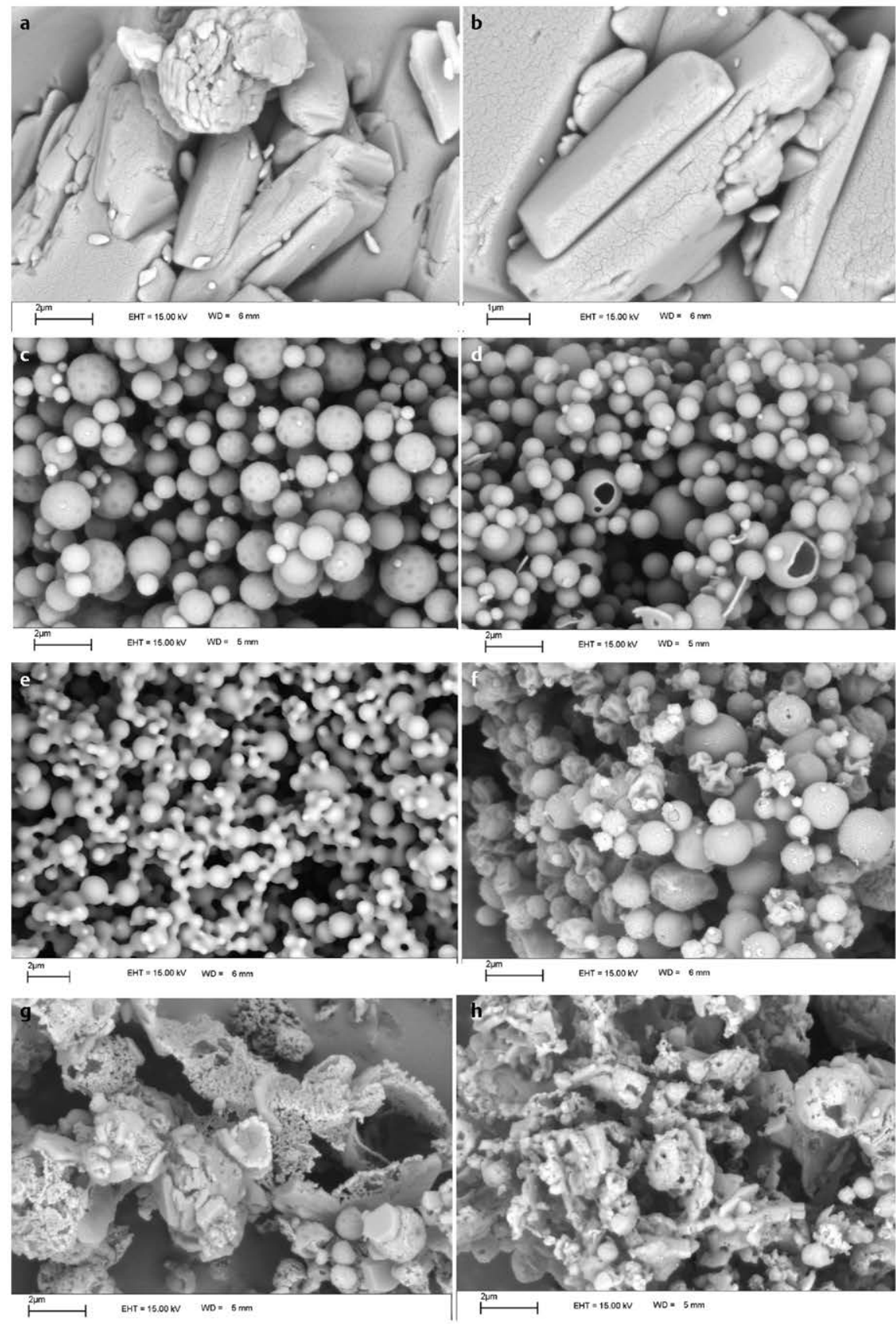

Fig. 1 Scanning electron microscopic images of a pure Ketotifen, $\mathbf{b}$ spray dried Ketotifen, and Formulations of c F1, d F2, e F3, f F4-1, g F4-2 and h F4-3. 
ley Inhaler Testing Data Analysis Software (CITDAS, version 3.10). The MMAD is defined as the diameter at which $50 \%$ of the particles by mass are larger and $50 \%$ are smaller. FPF represents the percentage of emitted particles with an MMAD of $5 \mu \mathrm{m}$ or less estimating the fraction of particles expected to deposit deep within the lungs. Water:ethanol ( $1: 1 \mathrm{v} / \mathrm{v})$ was used to wash the NGI cups and the total mass of drug was quantified via UV spectroscopy method, where responses were linear in the range of $10-50 \mu \mathrm{g} / \mathrm{mL}\left(\lambda_{\max }=300 \mathrm{~nm}\right.$, $\left.r^{2}=0.9981\right)$.

\section{Results and Discussion}

\section{Particle size and morphology}

Spray drying turned the geometric and angled drug particles ( $\vee$ Fig. 1a, b) to the circle particles ( $\triangleright$ Fig. 1c). Besides, addition of ethanol resulted in the production of porous and light particles while increasing the ethanol percentage in the feed mixture caused adhesion of particle, decreasing particle size, and improving the uniformity of particles ( $\triangleright$ Fig. 1d, e). Finally, the addition of ammonium bicarbonate enhanced the porosity of particles and decreased the spherical-shaped particles ( $\mathbf{F i g . ~ 1 f , ~} \mathbf{g}, \mathbf{h}$ ). Overall, Formulation F2 led to producing the spherical, uniform, and porous particles.

\section{DSC and XRD analysis}

XRD and DSC analyses were performed to assess possible relevant modifications of crystallinity. $>$ Fig. 2 shows that the XRD pattern and DSC thermograms of pure Ketotifen and spray-dried formulation instantly after preparation and 18 months after preparation. Both experiments indicated that Ketotifen changes to an amorphous state in spray-dried powder. After 18 months, crystallites

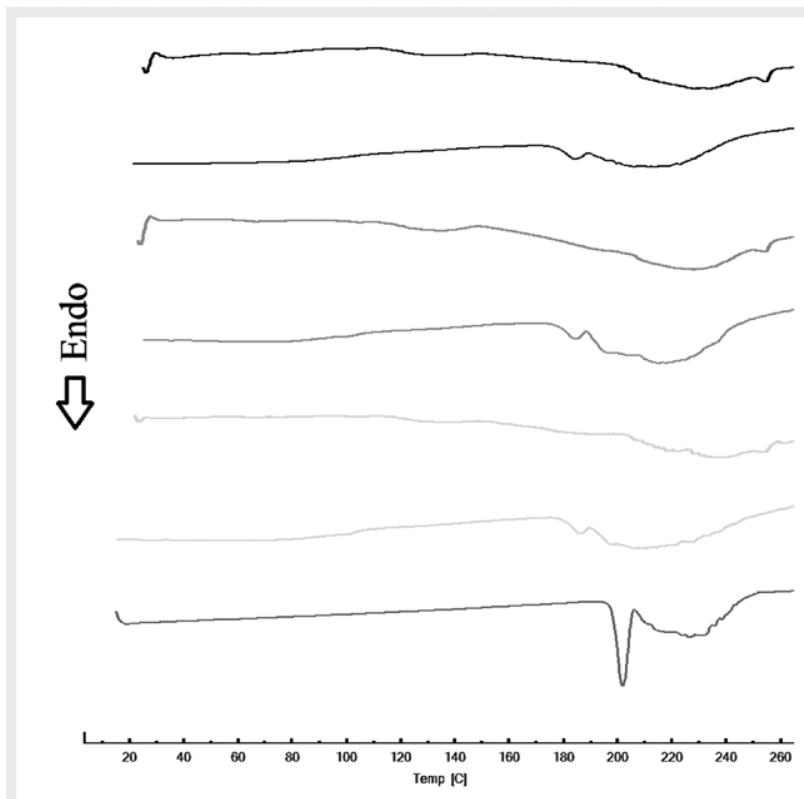

- Fig. 2 Differential scanning calorimetry thermograms a X-ray diffraction patterns $\mathbf{b}$ of pure Ketotifen and formulation instantly and 18 months after preparation. start to grow in the Ketotifen and its melting point decreases from 197 to $185^{\circ} \mathrm{C}$ ( $\mathbf{F i g}$. 2a). Usually, spray-drying process causes some changes in the crystalline structure of drug to an amorphous one. Afterward, the drug turns to the crystalline structure during the storage time, leading to a non-predictable drug performance. However, in this case, Ketotifen itself was amorphous from the beginning and no change happened after formulation process. Therefore, it guarantees the reproducible performance of formulated carrier free Ketotifen dry powder inhaler during the shelf life of dosage form. However, after 18 months, melting enthalpy decreased to $11.09 \mathrm{~J} / \mathrm{g}$ in a formulation containing ethanol in comparison with the formulations prepared by water $(13.96 \mathrm{~J} / \mathrm{g}$ ) and water:ethanol $(14.34 \mathrm{~J} / \mathrm{g})$. The lack of peaks associated with Ketotifen in spray-dried powders demonstrates the decreasing in the initial crystalline state of Ketotifen, which resulted in a better inhalation performance.

\section{FTIR spectroscopy}

Furthermore, DSC thermograms of formulation F4-1, containing ammonium bicarbonate in the formulation, showed an interference or coordination between Ketotifen and ammonium bicarbonate ( $\vee$ Fig. 3a), which needs to be further analyzed with FTIR ( $>$ Fig. 3b). $\triangleright$ Fig. 3 b shows the FTIR peaks of Ketotifen, ammonium bicarbonate, and spray-dried formulation of Ketotifen with ammonium bicarbonate in the 1:1 ratio (F4-1), which are used to analyze the interactions between the drug and excipient. Changing the peaks locations belonging to $\mathrm{C}-\mathrm{N}\left(1254\right.$ to $\left.1,281 \mathrm{~cm}^{-1}\right), \mathrm{C}=\mathrm{C}$ (1394 to $1402 \mathrm{~cm}^{-1}$ ), $\mathrm{C}=\mathrm{O}$ (1717 to $1701 \mathrm{~cm}^{-1}$ ) and $\mathrm{O}-\mathrm{H}$ (2482 to $2368 \mathrm{~cm}^{-1}$ ) demonstrate the interaction between Ketotifen and ammonium bicarbonate.

\section{Density measurement and flow property analysis}

Powder flow property was studied by determination of bulk and tap density. Results showed that spray drying improved the flowability in the case of all formulations in the following order: F1 > F2 > F3. Results also showed that addition of ethanol decreased the true density; however, $\mathrm{HR}$ and $\mathrm{Cl}$ values indicated that flowability was decreased probably due to the increasing powder adhesion ( $\triangleright$ Table 2 ). Therefore, flowability was found as a critical physical property responsible for the aerosolization performance.

\section{Evaluation of inhalation performance with $\mathrm{NGI}$}

Pulmonary delivery is considered as a rapid clinical response and the capability to bypass therapeutic barriers including poor gastrointestinal absorption and first-pass hepatic metabolism since it reduces dose and side effects [21]. For a suitable DPI formulation, developed particles should have a mean aerodynamic size below $5 \mu \mathrm{m}$ [16]. After preparation of the nanoparticles, the next phase is the control of aggregation for the preparation of respirable particles $(1-5 \mu \mathrm{m})$. In this connection, the spray-drying technique has been introduced as a suitable method for preparation of particles in this range. Spray drying is a one-step and low-cost pharmaceutical process extensively used in the preparation of dry powder formulations since it offers a number of potential benefits over lyophilization method and development of uniformly sized particles with preferred aerosolization properties [22]. Local delivery of Ketotifen 
to the lungs via nanoparticles is a novel therapeutic option for the use of Ketotifen in asthma. Inhalation performance of pure Ketotifen and spray-dried powder of Ketotifen was compared using a Next Generation Impactor (NGI) apparatus. Due to the flexibility in use and high productivity, NGls (a new impactor type specifically designed for testing pharmaceutical inhalers using the very newest and modern designed theory in 1997) have been used as the most popular testing machine within many inhaler research laboratories. NGI was launched in 2000 and was subsequently accept-

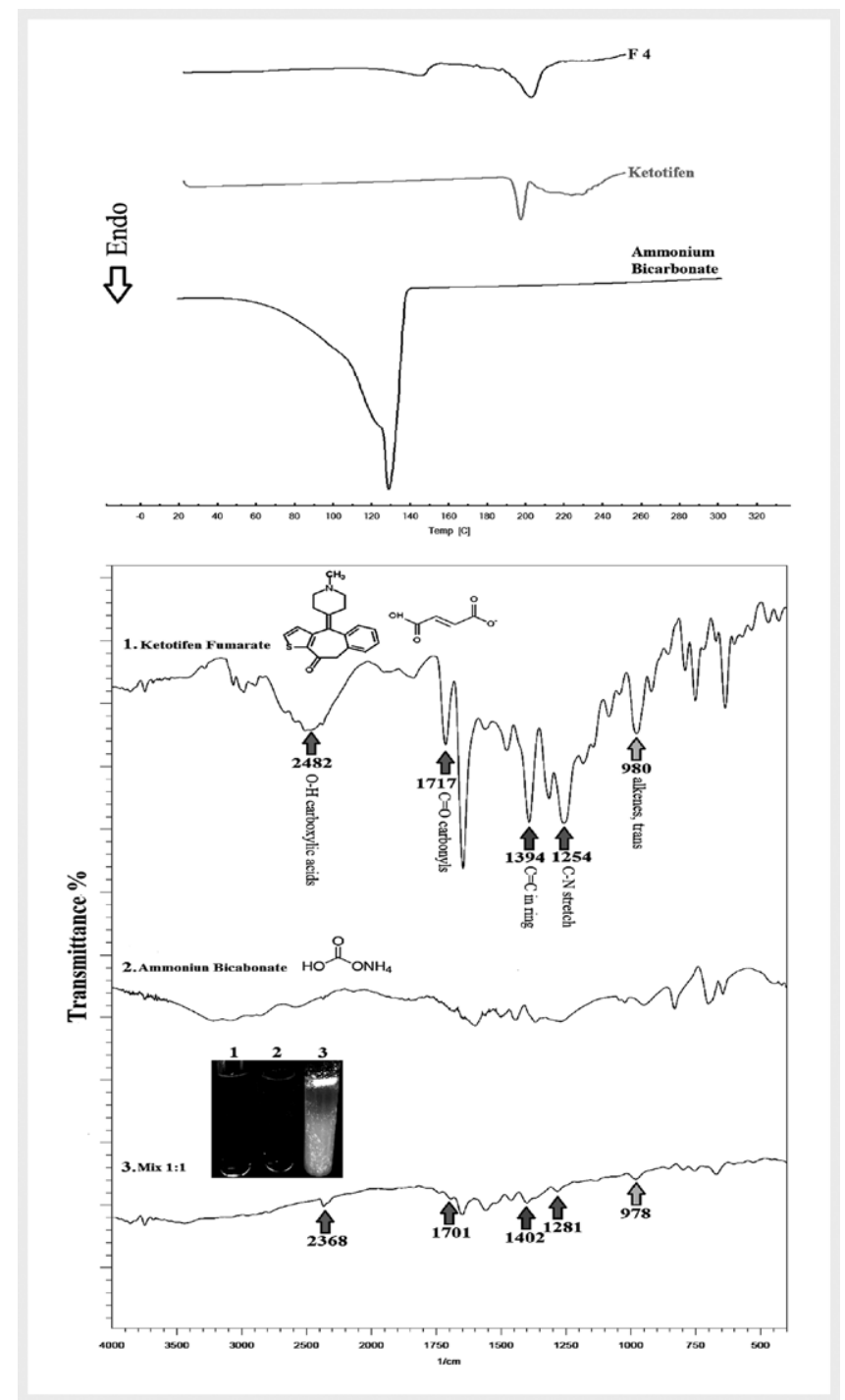

- Fig. 3 Differential scanning calorimetry thermograms a and Fourier Transform Infrared spectroscopy b of pure Ketotifen, ammonium bicarbonate and sprayed dried Formulation of them (F4-1). ed in the European Pharmacopeia as Apparatus E and in the United States Pharmacopeia as Apparatuses 5 and 6 in 2005, respectively. The details of Ketotifen deposition in different stages of $\mathrm{NGI}$ are presented in $>$ Fig. 4. Effects of the different solvent mixture in the aerosolization performance of formulations resulted from $>$ Fig. 4 are summarized in $>$ Table 3.

Morphology of drug particles seems to be the most important issue in the carrier free DPI formulation to avoid drug aggregation during aerosolization step. The degree of dispersion in a log-normally distributed aerosol is characterized by the GSD. A larger GSD implies a longer large particle size tail in the distribution [23]. GSD for a well-functioning stage should ideally be less than 1.2 while it would be 1.0 for an ideal size fractionator $[9,24]$. The ideal size for a therapeutic particle is not known exactly but it is assumed that the MMAD should not exceed $5 \mu \mathrm{m}$ to pass into the tracheobronchial tree and smaller airways if the peripheral deposition is required [25]. Results showed that in all cases spray drying with any solvent improve the aerosolization properties in comparison with micronized Ketotifen. Furthermore, formulation F2 exhibited an MMAD of $3.76 \pm 0.32 \mu \mathrm{m}$ and a higher FPF $(24.84 \pm 3.08)$ in comparison to the other formulations. Considering the other studies and all results obtained by flowability test, SEM, XRD, DSC, FTIR, and evaluation of inhalation performance experiments, Formulation F2 was found as the best formulation that could be used for further in vivo research.

\section{Conclusions}

The use of aerosol therapy for lungs drug delivery in asthma and chronic obstructive pulmonary diseases has been considerably in-

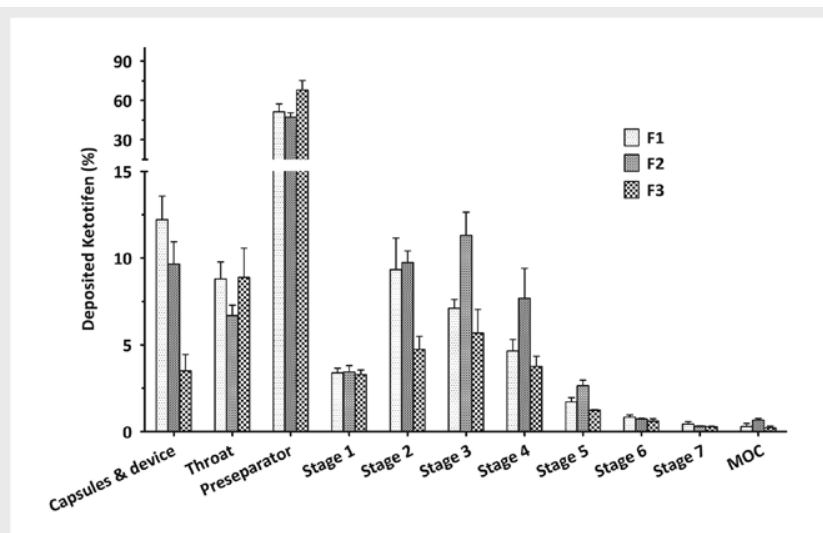

Fig. 4 Comparison of the amount of deposited Ketotifen of injected spray-dried formulations in various stages of $\mathrm{NGl}$ (data presented as mean $\pm S D, n=3$ ).

- Table 2 True, bulk and tap density as well as Hausner ratio (HR) and compressibility index (CI) of bulk and spray-dried formulations of Ketotifen.

\begin{tabular}{|l|c|c|c|c|c|}
\hline Formulation & True density & Bulk density & Tap density & HR & Cl (\%) \\
\hline F1 & $1.34 \pm 0.003$ & $0.17 \pm 0.01$ & $0.22 \pm 0.01$ & $1.25 \pm 0.15$ & $20.00 \pm 0.7$ \\
\hline F2 & $1.34 \pm 0.007$ & $0.12 \pm 0.02$ & $0.16 \pm 0.01$ & $1.36 \pm 0.17$ & $26.67 \pm 1.1$ \\
\hline F3 & $1.33 \pm 0.001$ & $0.21 \pm 0.01$ & $0.30 \pm 0.02$ & $1.44 \pm 0.11$ & $30.80 \pm 2.0$ \\
\hline Ketotifen & $1.35 \pm 0.001$ & $0.33 \pm 0.02$ & $0.49 \pm 0.02$ & $1.50 \pm 0.19$ & $33.33 \pm 3.6$ \\
\hline
\end{tabular}


- Table 3 Effects of different solvent mixture in the aerosolization performance of formulations.

\begin{tabular}{|l|c|c|c|c|}
\hline Parameters & Ketotifen & eF1 & F2 & F3 \\
\hline aFPD (mg) & $0.87 \pm 0.13$ & $3.02 \pm 0.82$ & $3.98 \pm 0.95$ & $2.63 \pm 0.44$ \\
\hline bFPF (\%) & $5.73 \pm 1.15$ & $19.8 \pm 2.27$ & $24.84 \pm 3.08$ & $13.33 \pm 3.0$ \\
\hline 'MMAD $(\boldsymbol{\mu m})$ & $4.00 \pm 0.83$ & $4.00 \pm 0.83$ & $3.76 \pm 0.32$ & $3.78 \pm 0.46$ \\
\hline dGSD & $2.09 \pm 0.065$ & $1.84 \pm 0.26$ & $1.83 \pm 0.012$ & $2.03 \pm 0.06$ \\
\hline $\begin{array}{l}\text { aFine Particle Dose; bFine Particle Fraction; CMass Median Aerodynamic Diameter; dGeometric Standard Deviation; eF1, F2 and F3 were prepared by } \\
\text { spray drying of Ktotifen in Water, Water:ethanol and ethanol, respectively }\end{array}$ \\
\hline
\end{tabular}

creased in the recent years. Compared to other dosage forms, DPIs have been received much attention in the recent decades and the performance of DPI inhalers has been improved in drug and carrier particle engineering. The main objective with inhalers is to obtain powders with high pulmonary deposition, which can be highly affected by the physicochemical characteristics of drugs. To achieve this objective, suitable solution and optimization of spray drying conditions are needed. Data from the present study indicate that the simple, one-step, low-cost, and rapid-acting characteristics of spray drying make it a promising method for producing the DPI formulation of Ketotifen on an industrial scale [16, 17, 26]. The results of this study introduced the potential application of DPIs for lung delivery of various drugs.

\section{Acknowledgments}

This paper was extracted from Pharm.D. thesis No. 3790 that was submitted to the Faculty of Pharmacy of Tabriz University of Medical Sciences and financially supported by Drug Applied Research Center of the same university (Grant No. 58359).

\section{Conflict of Interest}

The authors report no conflicts of interest.

\section{References}

[1] Russo P, Santoro A, Prota L et al. Development and investigation of dry powder inhalers for cystic fibrosis. In: Demir Sezer A. ed. Recent Advances in Novel Drug Carrier Systems. InTech 2012: 17-38

[2] Rojanarat W, Changsan N, Tawithong E et al. Isoniazid proliposome powders for inhalation-preparation, characterization and cell culture studies. International Journal of Molecular Sciences 2011; 12: 4414-4434

[3] Rahimpour Y, Hamishehkar H. Lactose engineering for better performance in dry powder inhalers. Advanced Pharmaceutical Bulletin 2012; 2: 183

[4] Hamishehkar H, Emami J, Najafabadi AR et al. Influence of carrier particle size, carrier ratio and addition of fine ternary particles on the dry powder inhalation performance of insulin-loaded PLGA microcapsules. Powder Technology. 2010; 201: 289-295

[5] Hamishehkar H, Emami J, Najafabadi AR et al. Effect of carrier morphology and surface characteristics on the development of respirable PLGA microcapsules for sustained-release pulmonary delivery of insulin. International Journal of Pharmaceutics. 2010; 389: 74-85
[6] Leonardi S, Coco A, Del Giudice MM et al. The airway epithelium dysfunction in the pathogenesis of asthma: The evidence. Health 2013; 5: 331-338

[7] Sung JC, Pulliam BL, Edwards DA. Nanoparticles for drug delivery to the lungs. Trends in Biotechnology 2007; 25: 563-570

[8] Ugwoke MI, Vereyken IJ, Luessen H. Microparticles and Liposomes as Pulmonary Drug Delivery Systems: What Are the Recent Trends? Chapter VI. 2007; 308-377

[9] Emami J, Hamishehkar H, Najafabadi AR et al. Particle size design of PLGA microspheres for potential pulmonary drug delivery using response surface methodology. Journal of Microencapsulation 2009; 26: $1-8$

[10] Emami J, Hamishehkar H, Najafabadi AR et al. A novel approach to prepare insulin-loaded poly (lactic-co-glycolic acid) microcapsules and the protein stability study. Journal of Pharmaceutical Sciences. 2009; 98: 1712-1731

[11] Ghanbarzadeh S, Valizadeh H, Yaqoubi S et al. Application of Spray Drying Technique for Flowability enhancement of Divalproex Sodium. Drug Research 2018; 68: 168-173

[12] Chen X, Zhong D, Liu D et al. Determination of ketotifen and its conjugated metabolite in human plasma by liquid chromatography/ tandem mass spectrometry: Application to a pharmacokinetic study. Rapid Communications in Mass Spectrometry 2003; 17: 2459-2463

[13] Petrasch S, Van Tits LJH, Motulsky HJ et al. Effects of ketotifen on human lymphocytes in vitro and in vivo. Arzneimittel-Forschung/Drug Research 1993; 43: 904-908

[14] Unno K, Ozaki T, Mohammad S et al. First and second generation $\mathrm{H} 1$ histamine receptor antagonists produce different sleep-inducing profiles in rats. European journal of pharmacology 2012; 683: 179-185

[15] Misawa M, Kanai Y, Chiba Y. Effects of the new antiallergic drug epinastine and ketotifen on repeated antigen challenge-induced airway hyperresponsiveness in rats. Arzneimittel-Forschung/Drug Research 1991; 41: 1277-1280

[16] Joshi M, Misra A. Dry powder inhalation of liposomal Ketotifen fumarate: Formulation and characterization. Int J Pharm. 2001; 223: $15-27$

[17] Joshi M, Misra A. Disposition kinetics of ketotifen from liposomal dry powder for inhalation in rat lung. Clinical and experimental pharmacology \& physiology 2003; 30: 153-156

[18] Haque S, Whittaker M, McIntosh MP et al. A comparison of the lung clearance kinetics of solid lipid nanoparticles and liposomes by following the $3 \mathrm{H}$-labelled structural lipids after pulmonary delivery in rats. European Journal of Pharmaceutics and Biopharmaceutics. 2018; 125: $1-12$

[19] Ullah N, Noreen S, Tehreem K et al. Liposome as nanocarrier: Site targeted delivery in lung cancer. Asian Pacific Journal of Tropical Disease 2017; 7: 502-512

[20] United States Pharmacopeia 35 - National Formulary 30, General Chapter 601 (2015) 
[21] Claus S, Weiler C, Schiewe J et al. How can we bring high drug doses to the lung? Euro J Pharma Biopharm 2014; 86: 1-6

[22] Sung JC, Pulliam BL, Edwards DA. Nanoparticles for drug delivery to the lungs. Trends biotech 2007; 25: 563-570

[23] Musante C, Schroeter J, Rosati J et al. Factors affecting the deposition of inhaled porous drug particles. J Pharm Sci 2002; 91: $1580-1590$
[24] Marple V, Olson B, Santhanakrishnan K et al. Next generation pharmaceutical impactor. Part II : Calibration. J Aerosol Med 2003; 16: 301-324

[25] Newman S, Clarke S. Therapeutic aerosols 1-physical and practical considerations. Thorax 1983; 38: 881-886

[26] Elsayed MM, Abdallah O, Naggar $V$ et al. Deformable liposomes and ethosomes as carriers for skin delivery of ketotifen. Die Pharmazie-An International Journal of Pharmaceutical Sciences 2007; 62: 133-137 